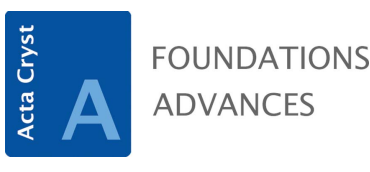

ISSN 2053-2733

Received 28 May 2019

Accepted 25 October 2019

Edited by D. A. Keen, STFC Rutherford Appleton Laboratory, UK

Keywords: stacking faults; pair distribution function; Debye function analysis; close-packed metals; cobalt.

Supporting information: this article has supporting information at journals.iucr.org/a

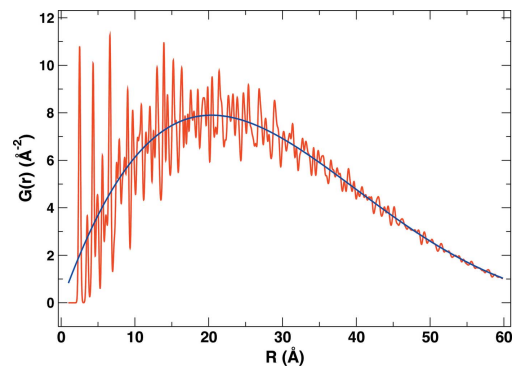

C 2020 International Union of Crystallography

\section{A real-space approach to the analysis of stacking faults in close-packed metals: $G(r)$ modelling and $Q$-space feedback}

\author{
Alessandro Longo, ${ }^{\mathrm{a}, \mathrm{b}}$ Francesco Giannici, ${ }^{\mathrm{c}}$ Luisa Sciortino ${ }^{\mathrm{c}}$ and Antonino \\ Martorana $^{\mathrm{C} *}$
}

${ }^{\mathbf{a} X M I}$, Department of Chemistry, Ghent University, Krijgslaan 281 S12, Ghent, East Flanders, 9000, Belgium, ${ }^{\mathbf{b}}$ Istituto per lo Studio dei Materiali Nanostrutturati (ISMN)-CNR, UOS Palermo, Via Ugo La Malfa, 153, Palermo, 90146, Italy, and ${ }^{\mathbf{c}}$ Dipartimento di Fisica e Chimica, Università di Palermo, Viale delle Scienze, Palermo, I-90128, Italy. ${ }^{*}$ Correspondence e-mail: antonino.martorana@unipa.it

An $R$-space approach to the simulation and fitting of a structural model to the experimental pair distribution function is described, to investigate the structural disorder (distance distribution and stacking faults) in close-packed metals. This is carried out by transferring the Debye function analysis into $R$ space and simulating the low-angle and high-angle truncation for the evaluation of the relevant Fourier transform. The strengths and weaknesses of the $R$-space approach with respect to the usual $Q$-space approach are discussed.

\section{Introduction}

The study of structural disorder in metals was the object of pioneering work in the early decades of the past century (Wilson, 1942; Warren \& Averbach, 1952; Mitra \& Hadler, 1964). The classical book of Warren (1969) reviews this topic.

This paper demonstrates the possibility of simulating the pair distribution function (PDF) of a metal with stacking fault disorder. The structural analysis of nanosized metal samples has been the object of several studies carried out by making use of the PDF and fitting of models to experimental data (Page et al., 2004; Rocha et al., 2009; Dmowski et al., 2010; Poulain et al., 2016; Hong et al., 2016; Li et al., 2018). It goes without saying that the $R$ and $Q$ approaches should in principle be consistent with each other, if the full range of data was available in both spaces. A nice discussion, in relation to this concern, is given in the book by Egami \& Billinge (2003, Section 6.3). Among the peculiarities of $R$-space analysis, the possibility of its limitation to a given $R$-space interval could give useful information about the dependence of structural features on the distance range considered. Moreover, the width of the PDF peaks depends essentially on the distance distribution between pairs of scattering centres, while their height is determined by the number and also of course, in the case of polyatomic samples, by the type of neighbours; in contrast, particle size and structural disorder both contribute to peak broadening in $Q$ space, and disentangling the two effects could result in strong correlation between size and disorder parameters, in particular when dealing with nanostructured samples. Conversely, the $Q$ approach could be important for the structural analysis of bulk samples, and in 
particular to correlate peak width to particle shape and size distribution. Thus, joint $Q$ and $R$ refinements could provide important and complementary structural insight in material science (Egami \& Billinge, 2003, Section 6.3).

Among the most striking examples of the occurrence of stacking fault disorder in close-packed metals is the case of cobalt. Its diffraction pattern is characterized by the ubiquitous presence, irrespective of particle size and synthesis route, of both the face-centred cubic (f.c.c.) and hexagonal closepacked (h.c.p.) signatures: these constitute clear evidence of the occurrence of complex patterns of stacking faults in this metal, as observed by several authors (more recently, Sokalski et al., 2011; Longo et al., 2014; Sławiński et al., 2018; Li et al., 2018). More generally, stacking fault disorder is recognized in several close-packed metals, even if in a less evident way than in cobalt (Yu et al., 2016; Bertolotti et al., 2016; Dupraz et al., 2015; Longo \& Martorana, 2008). In the studies concerning the applications of cobalt as a component of catalytic systems or as a magnetic material, the structural analysis is often performed on a qualitative basis by merely recognizing the contribution of f.c.c. and h.c.p. phases; this approach could be ineffective, or even misleading, for establishing reliable structure-property correlations. This issue can be particularly relevant for the magnetic properties, since it is well recognized that stacking faults have a strong influence on the magnetic anisotropy of cobalt and cobalt-based materials (Pousthomis et al., 2015; Kha et al., 2017), but also the influence of structural disorder on the catalytic properties of cobalt deserves an indepth investigation (Gnanamani et al., 2012). We have recently proposed a structural model that is able to simulate the main X-ray diffraction (XRD) features of samples of nanostructured cobalt, using a Markovian sequence of closepacked structural layers (Longo et al., 2014). The model allows for the probability that an atomic layer influences the closepacked arrangement of the next atomic layers, up to the fourth neighbour, and it is based on the matrix formalism proposed by Kakinoki (1967). A complete fitting of the XRD pattern was achieved by modelling the shape (roughly spherical) and the size distribution (log-normal) of the coherent diffraction domains, while the structural analysis was integrated and substantiated by the short-range information obtained by $\mathrm{X}$-ray absorption spectroscopy. In this paper, we outline an approach to the simulation and fitting of a structural model to the experimental PDF of close-packed metals. Aiming specifically at testing its reliability and limits for the analysis of real samples, the $R$-space approach was applied to the PDF data of a simulated nanosized sample and to the data of a real sample constituted of large Co crystallites.

The paper is organized as follows: the next section (Section 2) outlines the main equations of the model; Section 3 describes some details of the fitting runs, in order to illustrate the limits and peculiarities of the approach, also in relation to a reciprocal-space approach; Section 4 deals with the consistency of the $Q$ - and $R$-space analyses; in the last section (Section 5) the conclusions and a summary of the pros and cons of the $R$-space approach compared with the usual reciprocal-space approach are reported.

\section{Theory}

\subsection{Model $\mathrm{G}(r)$ of a monoatomic isotropic sample}

The powder intensity of an isotropic assembly of identical monoatomic crystallites is given by

$$
I(Q)=f^{2}\left[N+\sum_{\substack{n, m \\ n \neq m}}^{N} \frac{\sin \left(Q d_{n m}\right)}{Q d_{n m}}\right]
$$

where $f$ is the scattering cross section, $N$ is the number of atoms of each crystallite, $d_{n m}$ is the distance between the $n$th and the $m$ th atoms, and $Q=4 \pi \sin (\theta) / \lambda$.

Taking into account the fact that several interatomic distances can be coincident, the double sum of equation (1) can be rearranged as

$$
I(Q)=f^{2}\left[N+\sum_{d_{n} \neq 0} \mu_{d_{n}} \frac{\sin \left(Q d_{n}\right)}{Q d_{n}}\right]
$$

where $\mu_{d_{n}}$ represents the multiplicity of the $n$th distance $d_{n}$ between atom pairs; the sum is extended to all the different distances.

The cross terms normalized per atom are then given by

$$
i(Q)=\left[\frac{I(Q)}{N f^{2}}-1\right]=\frac{1}{N} \sum_{d_{n} \neq 0} \frac{\mu_{d_{n}}}{d_{n}} \frac{\sin \left(Q d_{n}\right)}{Q}
$$

and the sine Fourier transform (FT) of $Q i(Q)$ yields the related reduced PDF (Cervellino et al., 2006):

$$
\begin{aligned}
G(r) & =\frac{2}{\pi} \int_{0}^{\infty} Q i(Q) \sin (Q r) \mathrm{d} Q \\
& =\frac{1}{N} \sum_{d_{n} \neq 0} \frac{\mu_{d_{n}}}{d_{n}}\left[\delta\left(r-d_{n}\right)-\delta\left(r+d_{n}\right)\right] .
\end{aligned}
$$

Equation (4) is an array of delta functions, involving infinitely narrow distributions of the distance between pairs of scatterers. In the real cases, a finite-width distribution of distances is originated by thermal motion and/or by static disorder. In the simplest case of uncorrelated distance distribution, equation (4) is modified as

$$
G(r)=\Delta(r) * \frac{1}{N} \sum_{d_{n} \neq 0} \frac{\mu_{d_{n}}}{d_{n}}\left[\delta\left(r-d_{n}\right)-\delta\left(r+d_{n}\right)\right]
$$

where $\Delta$ is a finite-width distribution, typically a Gaussian:

$$
\Delta(r)=\mathrm{FT}\left[\exp \left(-\frac{\sigma^{2}}{2} Q^{2}\right)\right]
$$

and '*' stands for convolution.

More generally, distances can be spread as a function of the magnitude or even of the direction of interatomic vectors (Jeong et al., 1999; Longo \& Martorana, 2008), a typical outcome of the presence of microstrains in various materials:

$$
G(r)=\frac{1}{N} \sum_{d_{n} \neq 0} \frac{\mu_{d_{n}}}{d_{n}} \Delta_{d_{n}}(r) *\left[\delta\left(r-d_{n}\right)-\delta\left(r+d_{n}\right)\right] .
$$


Simulation of data truncation beyond a given $Q_{\max }$ can be achieved by multiplication of $Q i(Q)$ by a suitable window function (Proffen \& Billinge, 1999), which is taken into account by

$$
G(r)=\frac{1}{N} \sum_{d_{n} \neq 0} \frac{\mu_{d_{n}}}{d_{n}} \Gamma_{d_{n}}^{b_{0}}(r) *\left[\delta\left(r-d_{n}\right)-\delta\left(r+d_{n}\right)\right]
$$

where

$$
\Gamma_{d_{n}}^{b_{0}}(r)=\mathrm{FT}\left[S^{b_{0}} \cdot \Delta_{d_{n}}(Q)\right] .
$$

In equation (9) $\Delta_{d_{n}}(Q)$ stands for the Fourier transform of $\Delta_{d_{n}}(r)$ and $S^{b_{0}}$ for the step function in $Q$ space:

$$
S^{b_{0}}= \begin{cases}1 & \text { if }|Q| \leq b_{0} \\ 0 & \text { otherwise }\end{cases}
$$

where $b_{0}=Q_{\max }$ represents the highest $Q$-range limit for the sine FT calculation of equation (3).

The small-angle scattering, usually not acquired in PDF measurements, is otherwise present in the simulated patterns and, for fitting to experimental data, it should be removed. A good approximation is obtained by the equation proposed by Farrow \& Billinge (2009):

$$
G(r)=4 \pi r\left[\rho(r)-\rho_{0} \gamma_{0}(r)\right]
$$

where $\rho(r)$ is the $R$-space pair density, $\gamma_{0}(r)$ is the angleaveraged characteristic function and $\rho_{0}$ is the average number density. An example of a calculated pattern is reported in Fig. 1, where the small-angle contribution to the $G(r)$ due to a log-normal distribution of spherical scattering domains is shown.

\subsection{Stacking faults in close-packed metals}

In a disordered structure the interatomic distances in equation (8) are weighted by the respective probability of occurrence, as given by a suitable structural model. Here we report the $R$-space application of the model previously used (Longo et al., 2014) for the $Q$-space analysis of nanostructured

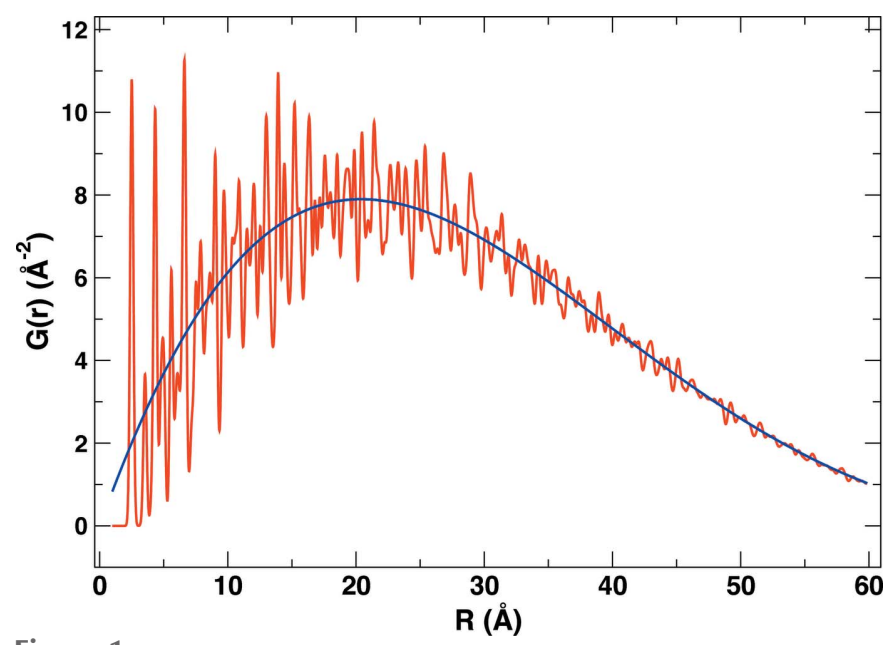

Figure 1

A calculated $G(r)$ of polydispersed spherical cobalt particles. The smallangle contribution is drawn in blue. cobalt samples, and based on the matrix formalism of Kakinoki (1967).

The faulted structure is a stack of (001) atomic layers labelled, as usual for close-packed structures, with $A, B, C$, according to the three allowed positions in the $(\mathbf{a}, \mathbf{b})$ hexagonal cell.

The sequence of atomic layers along $\mathbf{c}$ is modelled as a Markov chain of events. In the simplest case, each layer influences the occurrence of the next one, with the constraint that a sequence of equal layers $(A A$, say) is forbidden. A longer correlation range is allowed by assuming that the occurrence of an atomic layer depends, as a probabilistic event, on the block of preceding $s$ layers. The parameter $s$ is the so-called 'Reichweite' (Jagodzinski, 1949), defining how many previous layers influence the statistical occurrence of the next one in the stacking sequence. The number of distinct blocks is $S=3 \cdot 2^{s-1}$; for $s=2$, for instance, there are six blocks of two layers $(A B, A C, B C, B A, C A, C B)$, each block determining the probability of the next atomic layer: by symmetry, the $s=2$ model depends on one parameter, let us call it $\alpha$, for h.c.p. sequences ( $A B A$-type), and on $1-\alpha$ for $A B C$-type c.c.p. (cubic close-packed) sequences.

In the formalism of Kakinoki the probabilistic events are the $S$ blocks of $s$ atomic layers and the sequence probabilities are the elements of an $S \times S \mathbf{P}$ matrix, having the $S$ blocks at the head of the rows and columns. For $s=4$ the $S=24$ blocks are detailed in the supporting information. The $p_{\mu \nu}$ element $(\mu, \nu=1, \ldots, S)$ of the $\mathbf{P}$ matrix is different from zero if the $\mu$ th and $\nu$ th blocks are concatenated. This means that, for instance, $C \underline{B C A}$ (row heading) and $\underline{B C A B}$ (column heading) are concatenated if the last three layers of $C B C A$ are the same as the first three of $B C A B$. The corresponding element $p_{\mu \nu}$ of $\mathbf{P}$ gives the probability that $B$ (the last layer of $B C A B$ ) follows $A$ (the last layer of $C B C A$ ). So, the occurrence of $B$ after $A$ is statistically influenced by the $s=4 C B C A$ foregoing layers. This scheme gives the probabilities of sequences relative to the first neighbouring layers: the probabilities for $m$-neighbouring sequences are the elements $p_{\mu \nu}^{(m)}$ of $\mathbf{P}^{m}$, the $m$ th power of $\mathbf{P}$. The blocks can be classified according to the hexagonal (h) or cubic (c) close packing. So, $C B C A$ is an he sequence, $B C A B$ a cc, and so on. Then, by symmetry, the model depends on four independent probability parameters:

$\alpha$, probability of a cc_c concatenation, like $A B C A \_B$

$\beta$, probability of an hc_c concatenation, like $A B A C \_B$

$\gamma$, probability of an hh_c concatenation, like $A B A B \_C$

$\delta$, probability of a ch_c concatenation, like $A B C B \_A$.

The frequencies of occurrence of the blocks are

$$
\begin{aligned}
f_{\mathrm{cc}} & =\frac{\beta \gamma}{\Delta}, f_{\mathrm{hc}}=f_{\mathrm{ch}}=\frac{(1-\alpha) \gamma}{\Delta}, f_{\mathrm{hh}}=\frac{(1-\alpha)(1-\delta)}{\Delta} \\
\Delta & =6[(1-\alpha)(1-\delta)+2(1-\alpha) \gamma+\beta \gamma],
\end{aligned}
$$

obtained by solving the linear and homogeneous set of equations

$$
f_{\nu}=\sum_{\mu=1}^{S} f_{\mu} p_{\mu \nu}
$$


According to the 'parent stack' approach (Longo \& Martorana, 2008), the positions of the atomic layers in a stack can be horizontally shifted with respect to a fixed reference frame by the vectors:

$$
\mathbf{t}_{A}=-\frac{1}{3} \mathbf{a}-\frac{1}{3} \mathbf{b} ; \mathbf{t}_{B}=\frac{1}{3} \mathbf{a} ; \mathbf{t}_{C}=\frac{1}{3} \mathbf{b}
$$

and the relative position of two $m$-neighbouring atomic layers is

$$
\mathbf{t}_{\mu \nu}^{m}=m \mathbf{c}+\left(\mathbf{t}_{\mu}-\mathbf{t}_{\nu}\right) ; m=1,2, \ldots
$$

In equations (14), (15) $\mathbf{a}, \mathbf{b}, \mathbf{c}$ are the axes of the reference hexagonal cell. $\mu$ and $v$ run over all the $S=3 \cdot 2^{s-1}$ elementary sequences; $\mathbf{t}_{\mu}\left(\mathbf{t}_{v}\right)$ is the shift vector (of type $\mathbf{t}_{A}, \mathbf{t}_{B}$ or $\mathbf{t}_{C}$ ) of the last atomic layer of the $\mu$ th ( $\nu$ th) sequence. Two atoms belonging, respectively, to two $m$-neighbouring layers are then joined by the distance vector:

$$
\mathbf{t}_{\mu \nu}^{k l m}=k \mathbf{a}+l \mathbf{b}+\mathbf{t}_{\mu \nu}^{m}
$$

where $k, l, m$ are integers. The contribution of $\mathbf{t}_{\mu \nu}^{k l m}$ to the PDF, equation (8), is denoted as $G_{\mu \nu}^{k l m}$ and weighted by $\mu_{\mathbf{t}^{k l m}}$, the multiplicity of $\mathbf{t}^{k l m}=k \boldsymbol{a}+\boldsymbol{l} \boldsymbol{b}+m \boldsymbol{c}$, which is the number of times this distance is repeated in the parent stack (Longo \& Martorana, 2008):

$$
\mu_{\mathbf{t}^{k l m}}=\frac{1}{v} \int_{V} \mathrm{~d} \mathbf{r} \varphi\left(\mathbf{r}+\mathbf{t}^{k l m}\right) \varphi(\mathbf{r}) .
$$

In equation (17) $v$ is the volume per atom, $\varphi$ is the shape function, equal to 1 inside the particle and 0 outside; the integral is calculated over the volume, $V$, common to the particle and to its ghost shifted by $\mathbf{t}^{k l m}$. Then, the contribution to the PDF of all the pairs belonging to $m$-neighbouring layers joined by the $\mathbf{t}_{\mu \nu}^{m}$ vectors defined in equation (15) is

$$
\begin{aligned}
G_{\mu \nu}^{m}(r) & =\sum_{k l}^{\prime} G_{\mu \nu}^{k l m}(r) \\
& =\frac{1}{N} \sum_{k l}^{\prime} \frac{\mu_{\mathbf{t}^{k l m}}}{\left|\mathbf{t}_{\mu \nu}^{k l m}\right|} \Gamma_{\mathbf{t}_{\mu \nu}^{b_{0}}(r) *\left[\delta\left(r-\left|\mathbf{t}_{\mu \nu}^{k l m}\right|\right)-\delta\left(r+\left|\mathbf{t}_{\mu \nu}^{k l m}\right|\right)\right]}^{b^{k l m}},
\end{aligned}
$$

where the prime (') indicates exclusion of self terms.

According to the cited matrix formalism (Kakinoki, 1967), the average over all the $m$-neighbouring $(\mu, \nu)$ layers is then given by

$$
G^{m}(r)=\operatorname{tr}\left[\mathbf{A}^{(m)} \cdot \mathbf{P}^{(m)}\right]
$$

where $\left[\mathbf{A}^{(m)}\right]_{\mu \nu}=G_{\mu \nu}^{m} f_{v}$ and $\mathbf{P}^{(m)}=(\mathbf{P})^{m}$ is the $m$ th power of the probability matrix $\mathbf{P}$. The overall PDF is finally given by the sum over all the $m$-neighbours.

\section{Simulations and fitting}

\subsection{Simulated PDF data and fitting in $R$ space}

Aiming at testing the robustness and reliability of the $R$-space approach, we simulated the $Q$-space pattern of a nanostructured sample using:

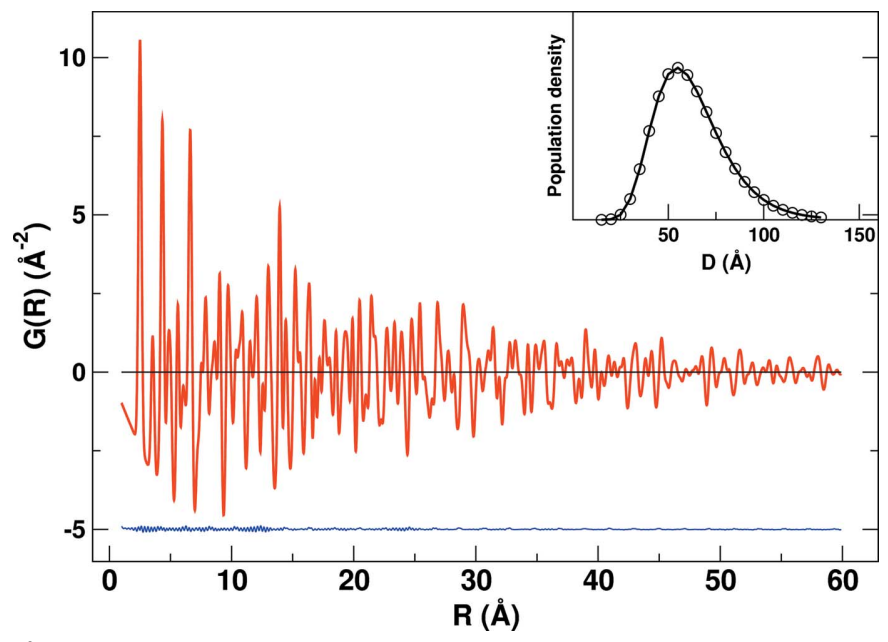

Figure 2

Best fitting (red) to the $G(R)$ data (see text). In the inset panel, the diameter distribution log-normal function is reported. The $G(R)$ data are omitted for the sake of clarity. The residual is plotted in blue.

(a) a log-normal size distribution of spherical particles drawn in Fig. 2 as a function of particle diameter;

(b) a Reichweite $s=4$ stacking fault model with probability values $\alpha=0.9677, \beta=0.0711, \gamma=0.1800, \delta=0.0400$, ensuring the presence of both the h.c.p. and c.c.p. distances;

(c) a $\Delta_{d_{n}}(Q)$, equation (9), defined as follows:

$$
\Delta_{d_{n}}(Q)=\exp \left[-Q^{2} \frac{1}{2}\left(\eta+\zeta d_{n}\right)\right]
$$

In equation (20) the parameter $\eta$ (input value $0.015 \AA^{2}$ ), accounting for thermal vibrations and uncorrelated static disorder, is related to the atomic temperature factor $B$ (Giacovazzo, 1992) by $B=\eta \pi^{2}$. It produces, as is well known, a damping of peak intensities for increasing $Q$, but does not affect peak width (Egami \& Billinge, 2003, Section 2.1.3); the corresponding Fourier transform involves in $R$ space a finite PDF peak width independent of distance, according to equation (5). On the other hand, $\zeta(=0.00019 \AA)$ governs the width of the Gaussian distribution of distances between $n$-neighbouring pairs as a function of their distance and can be related, in metals and other inorganic materials, to microstrains. The linear relation assumed in equation (20) involves an increasing uncertainty in the relative position of atom pairs as a function of $d_{n}$ (Hosemann \& Bagchi, 1962; Ruland, 1965; Longo et al., 2014). The effect of microstrains [equation (20)] in direct and reciprocal space is shown in Fig. S1 in the supporting information. A different dependence was proposed by Jeong et al. (1999), and will be discussed in the next section.

The $Q$ data were simulated with the Debye function approach (Longo et al., 2014) and an 'experimental' error was added, whose bandwidth is of the order of a typical synchrotron XRD experiment; the corresponding PDF was fitted by equations (7), (8) and (18) in the intervals $1<R<R_{\text {sup }} \AA$, with $R_{\text {sup }}=n \times 10, n=1,2, \ldots, 9$. The starting point for the fitting parameters was put far away from the known values. Fig. 2 shows the best fitting in the $1-60 \AA$ interval, while Fig. 3 shows 


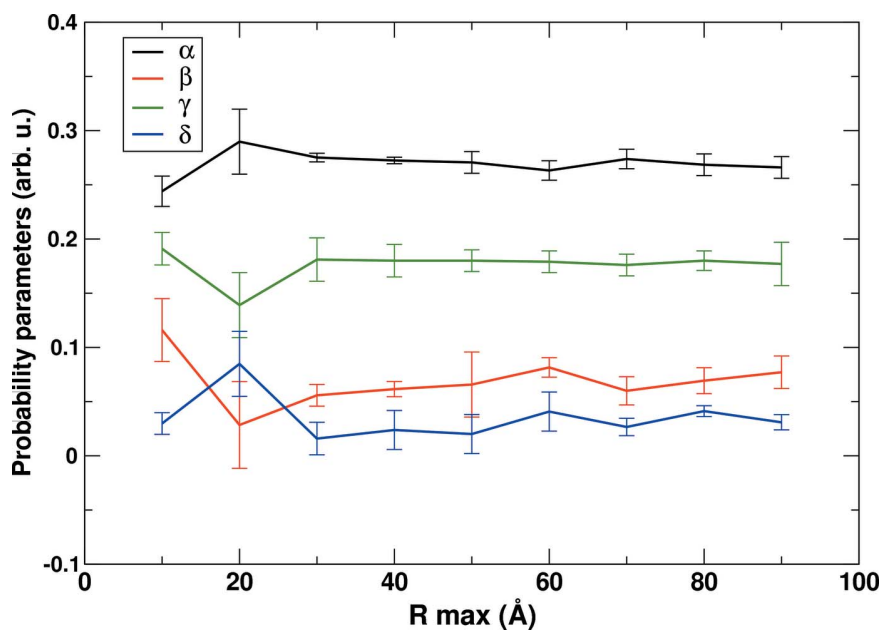

Figure 3

Fitted probability parameters as a function of the maximum $R$ value. For the sake of clarity, the value of $\alpha$ was offset by subtracting 0.7 .

the values of the optimized stacking fault parameters as a function of $R_{\text {sup. }}$

Fig. 3 shows some oscillation in the optimized parameters, depending on the range of the fitting interval and on the artificial error band introduced in the $Q$ pattern, with a tendency to converge to the 'true' values at large $R_{\text {sup. }}$ On the other hand, it is worth noticing that the overall frequencies of hexagonal, $f_{\mathrm{h}}=6\left(f_{\mathrm{hh}}+f_{\mathrm{ch}}\right)$, and cubic, $f_{\mathrm{c}}=6\left(f_{\mathrm{cc}}+f_{\mathrm{hc}}\right)$, sequences are very stable, capturing the 'true' values (respectively, 0.664 and 0.336 ) with a discrepancy of \pm 1 on the third digit for all the $R_{\text {sup }}$ values, but for the outlier $R_{\text {sup }}=20 \AA$ (yielding, anyway, not very different $f_{\mathrm{h}}=0.667$ and $f_{\mathrm{c}}=0.333$ ). The other parameters of the model, and in particular those governing the size distribution and the $(\eta, \zeta)$ pair of parameters giving the broadening of the $G(r)$ peaks, converge to the input values already at $R_{\text {sup }}=30 \AA$, that is at a distance corresponding to the average radius of the 'virtual sample'. Then, it looks like the $R$-space approach could allow a reliable determination of the overall stacking fault frequencies even over a limited distance range; this opportunity could be useful in view of the assessment of structure-property relationships in real samples. It should also be mentioned that the 'experimental' data used for testing the $R$ approach do not provide a length scale dependence of structural features; then, the behaviour reported in Fig. 3 depends only on the different amount of data provided to the fitting algorithm. However, it can be envisaged that in real samples the $R$ approach could give interesting information on the actual length scale dependence of all the structural features and, in particular, of the stacking fault parameters.

The issue of discriminating between different models is a tricky one, that can be treated by statistical approaches like the Hamilton's test (Hamilton, 1965). Taking advantage of the fact that the 'experimental' data are known in detail, the typical situation of an overparameterization of the structural model was reproduced, using a $s=4$ model to fit data corresponding to $s=3$. The simulated $G(r)$ relative to a Reichweite $s=3$ model depends on two probability parameters: $\alpha_{\mathrm{III}}$,

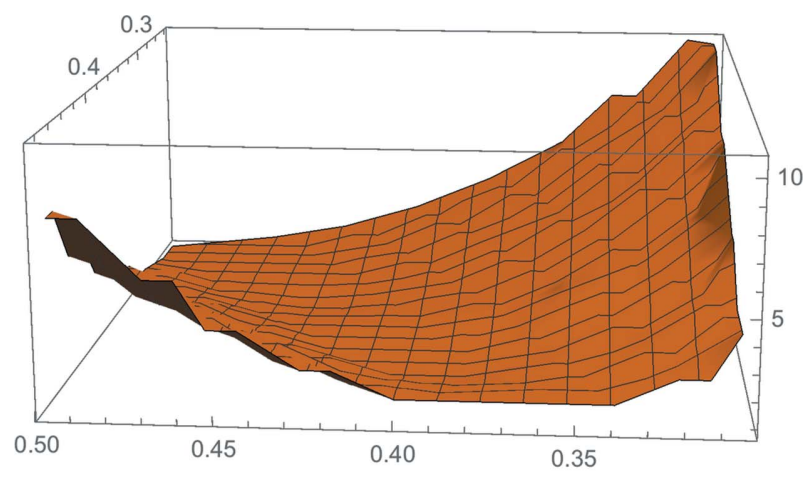

(a)

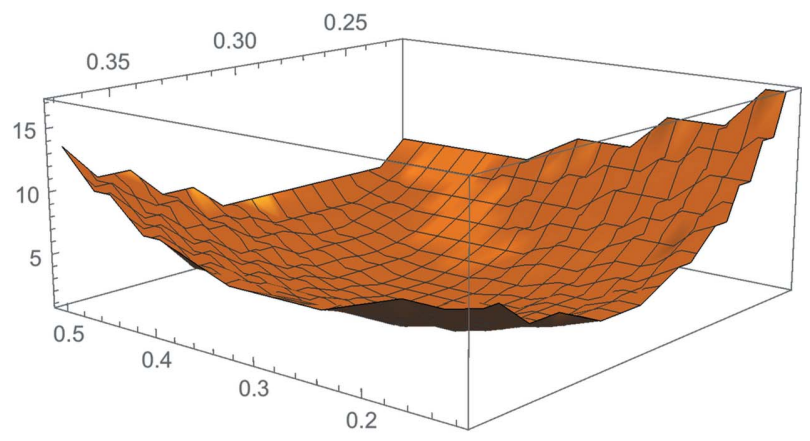

(b)

Figure 4

Behaviour of $\chi^{2}$ as a function of the pair of parameters $(\alpha, \beta)(a)$ and of the pair $(\gamma, \delta)(b)$ in the neighbourhood of the respective optimized values. The other parameters of the model are kept fixed at the converged values of the fitting run.

giving the probability of a c_c sequence (like $A B C \_A$ ), and $\beta_{\text {III }}$, for sequences h_c $\left(A B A \_C\right.$-type). The input values for the simulation of the 'experimental' data were $\alpha_{\mathrm{III}}=0.4$ and $\beta_{\mathrm{III}}=0.3$. However, the corresponding $G(r)$ pattern was analysed with a $s=4$ model, to check to what extent the $R$ approach is sensitive to the collapse of the $s=4$ model onto the $s=3$ case, that is, when $\alpha=\beta=\alpha_{\text {III }}$ and $\gamma=\delta=\beta_{\text {III }}$. Starting from a point with a definite $s=4$ character, the fitting run gave the optimized values $\alpha=0.35$ (8), $\beta=0.43$ (5), $\gamma=0.29$ (7) and $\delta=0.31(8)$, suggesting a fair capacity for discriminating between the $s=3$ and $s=4$ models. A deeper insight into this issue is provided by Figs. 4(a)-4(b), showing two scans of the $\chi^{2}$ in the space of the parameters: Fig. 4(a) shows the scan of $\chi^{2}$ as a function of the pair of parameters $(\alpha, \beta)$ around the ideal $(0.4,0.4)$ values, all the other parameters of the model $s=4$ being fixed at the minimum achieved in the above-described optimization run; Fig. 4(b) shows the corresponding space parameter scan involving the pair $(\gamma, \delta)$. While the variation of the latter pair of parameters shows a reasonably well defined minimum of $\chi^{2}$, the dependence of $\chi^{2}$ on the $(\alpha, \beta)$ pair shows a long shallow valley that is indicative, in this specific case, of the drawback of overparameterization.

\subsection{Analysis of a real bulk sample}

The model described in Section 2 was applied to the analysis of the X-ray diffraction data recorded on two different samples: (i) B1, bulk cobalt powder (Aldrich); (ii) G2, 


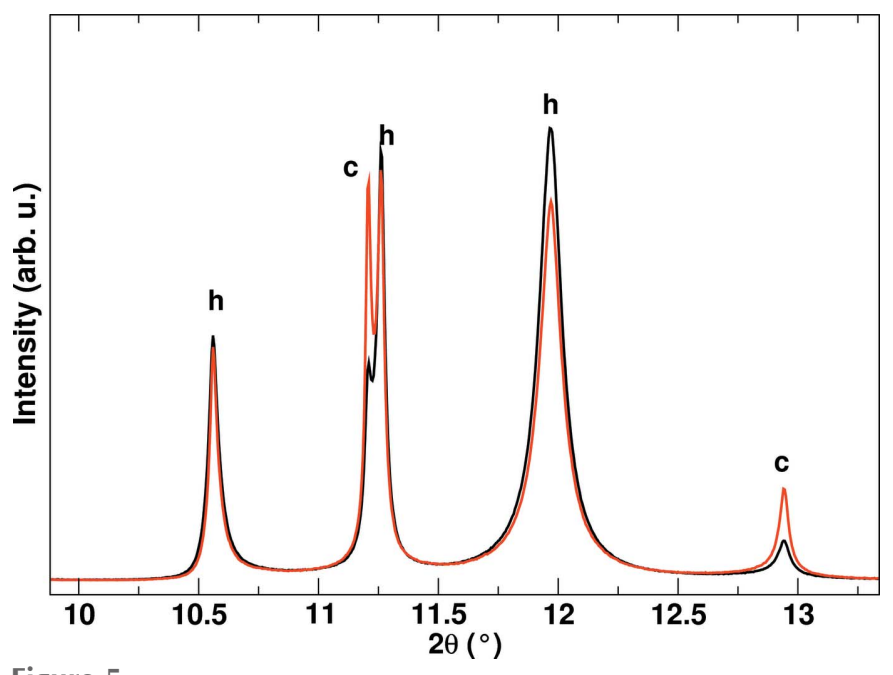

Figure 5

XRD pattern of bulk cobalt (red) and hand-milled cobalt (black) in the 10-13.2 $2^{\circ}$ region showing the presence of both h.c.p. and c.c.p. features. The h.c.p. and c.c.p. signatures are indicated as $h$ and c, respectively.

obtained by hand-grinding B1 for a few minutes in an agate mortar. The powder patterns were acquired in the $Q$ interval $1-21 \AA^{-1}$ at the ID22 beamline of the European Synchrotron Radiation Facility (Grenoble, France) in Debye-Scherrer geometry using $\lambda=0.4 \AA$ and a nine-channel Si 111 multianalyser stage (Hodeau et al., 1998).

The diffracted intensities reported in Fig. 5 show that the definitely mild grinding treatment produces a strong decrease in the cubic sequences which, as observed in the literature, could be due to the collapse of a metastable cubic phase formed during crystal growth (Meng et al., 2013). Besides the very large crystallite size, shown by the sharpness of the diffraction lines, traces of oxide phases are also present (see Fig. S1), e.g. $\mathrm{CoO}$ (main peaks at $\sim 9.3^{\circ}$ and $\sim 10.78^{\circ} 2 \theta$, the latter strongly overlapped with the metal) and $\mathrm{Co}_{3} \mathrm{O}_{4}$ (at $\sim 9.4^{\circ}, \sim 14.75^{\circ}$ and $\left.\sim 16.07^{\circ} 2 \theta\right)$. The $G(r)$ pattern was extracted by the pdfgetx 3 package (Juhás et al., 2013) and two distance ranges, $1 \leq d \leq d_{\max }=30 \AA$ and $1 \leq d \leq d_{\max }=60 \AA$, were selected for fitting. The model $G(r)$ was calculated for spherical crystallites, whose average diameter was determined by fitting the model to the data.

The model $G(r)$ for cobalt depends on: (i) the hexagonal lattice parameter $|\mathbf{a}|=|\mathbf{b}|$ and the distance $|\mathbf{c}|$ between neighbouring basal atomic layers; (ii) the four probability parameters $\alpha, \beta, \gamma, \delta$; (iii) an overall scale factor; (iv) the broadening of the $G(r)$ peaks. For the latter quantity, an inverse dependence on the distance, similar to that proposed by Jeong et al. (1999), was assumed:

$$
\sigma_{d_{n}}^{2}=\sigma_{0}^{2}-\frac{\sigma_{12}^{2}}{\left(d_{n}\right)^{1 / 2}}
$$

In equation (21) $d_{n}$ is the numerical value of the $n$th distance between pairs of atoms. This dependence is clearly different from the linear one assumed in equation (20). According to the latter, the uncertainty in the relative position of pairs increases linearly with distance, while the former reaches
Table 1

Fitting results in $R$ space.

\begin{tabular}{lll}
\hline & $\mathrm{B} 1$ & $\mathrm{G} 2$ \\
\hline$a(\AA)$ & $2.510(4)$ & $2.509(3)$ \\
$c(\AA)$ & $2.039(2)$ & $2.038(5)$ \\
$\alpha$ & $0.957(2)$ & $0.97(1)$ \\
$\beta$ & $0.17(1)$ & $0.071(2)$ \\
$\gamma$ & $0.087(6)$ & $0.08(2)$ \\
$\delta$ & $0.03(3)$ & $0.02(1)$ \\
$f_{\text {cc }}$ & 0.24 & 0.14 \\
$f_{\text {hc }}$ & 0.06 & 0.06 \\
$f_{\text {hh }}$ & 0.64 & 0.74 \\
$f_{\text {ch }}$ & 0.06 & 0.06 \\
$\sigma_{0}^{2}\left(\AA^{2}\right)$ & $0.016(1)$ & $0.018(1)$ \\
$\sigma_{12}^{2}\left(\AA^{2}\right)$ & $0.014(4)$ & $0.015(1)$ \\
$R_{\text {wp }}$ & 0.065 & 0.063 \\
\hline
\end{tabular}

asymptotically a constant value. As the fitting runs on B1 and G2 were carried out on a small interval of distances compared with the actual particle size, the functional dependence proposed by Jeong et al. (1999), quickly reaching the asymptotic behaviour, was preferred to the linear one of equation (20). Various fitting attempts showed that a not so swift attainment of the asymptotic value, as involved in Jeong's formula, was suitable and then, as an empirical trade-off, the dependence of equation (21) was adopted. The trend of equation (21) as a function of the optimized $\sigma_{0}^{2}$ and $\sigma_{12}^{2}$ parameters is shown in Fig. S3.

The fitting results in the $1-30 \AA$ distance interval are reported in Table 1 and Fig. 6.

The oxide phase was also taken into account in the formulation of the $R$-space model. However, its contribution is actually a minor one. Then, a possible model of a metal-oxide core-shell structure cannot be validated on the basis of experimental evidence. So, the $\mathrm{CoO}$ and $\mathrm{Co}_{3} \mathrm{O}_{4}$ structure was modelled at a low level, taking into account the crystallographic distances relevant to the two oxides, and only an average Gaussian broadening.

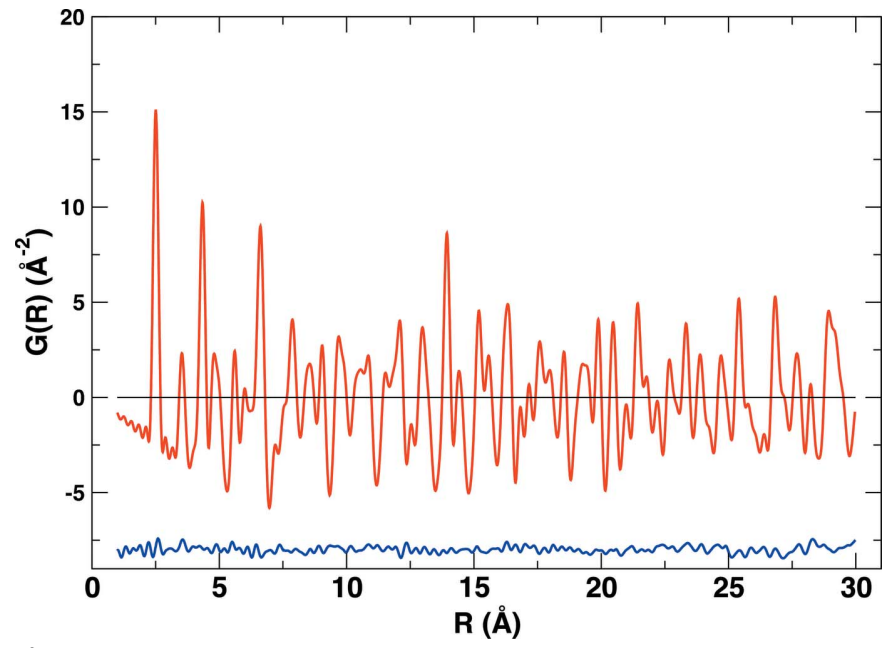

Figure 6

Best fitting (red) to the $G(R)$ data of sample G2 according to the model described in the text. The experimental data are omitted for the sake of clarity. The residual is plotted in blue. 


\section{Q-space feedback}

Simulation of the XRD pattern on the basis of the refined parameters reported in the previous section shows possible shortcomings that should be carefully evaluated when considering the $R$ approach.

Inspection of Fig. 7 shows that, although the model reproduces simultaneously all the peaks typical of both the f.c.c. and h.c.p. sequences, and the reflection-dependent broadening
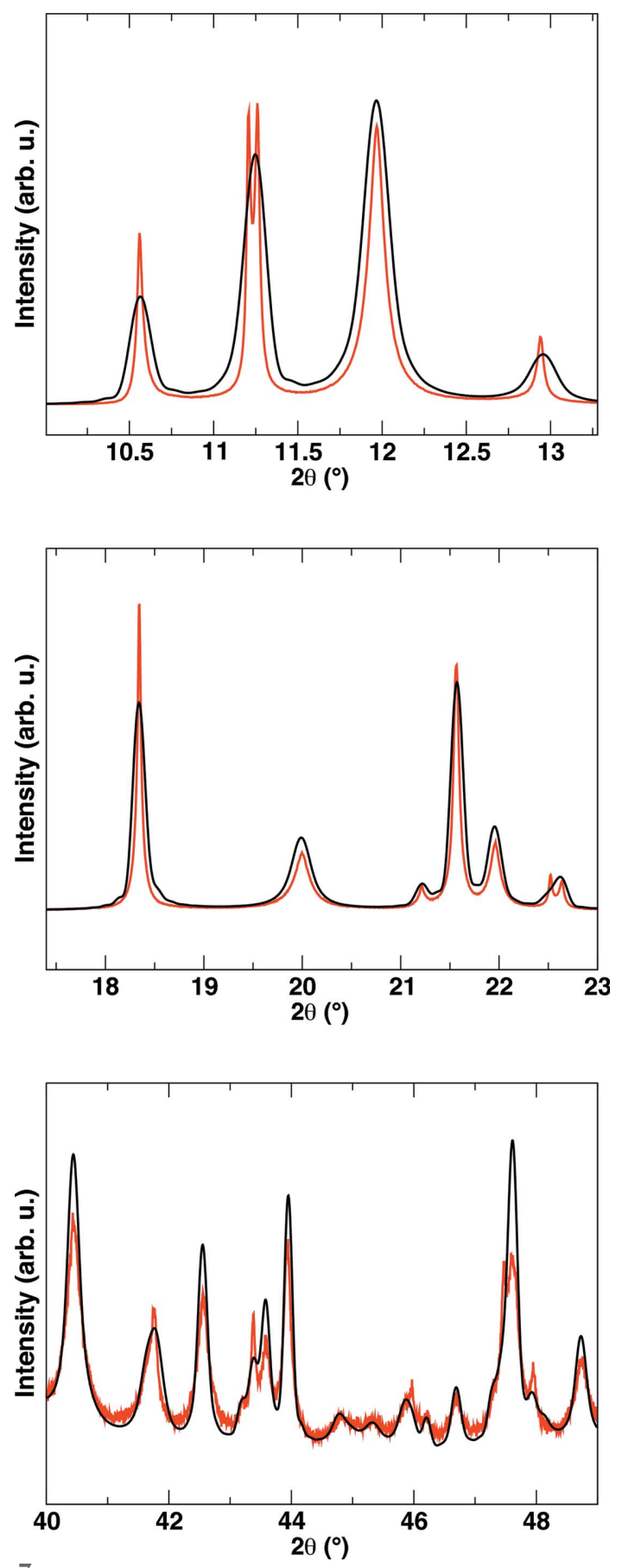

Figure 7

Experimental data of B1 cobalt sample (red) and simulated XRD pattern (black) according to the modelled parameters obtained from the PDF fitting. The intensities reported in the three frames are rescaled to show the wide-angle details of the XRD pattern. originated by stacking faults, the comparison between the calculated pattern and experimental data shows several sources of disagreement:

(i) Underestimation of particle size. This effect is particularly evident in the angular range $10-13.5^{\circ} 2 \theta$. In fact, the radii of the spherical crystallites obtained by fitting in the 1-30 range (96 and $83 \AA$, for $\mathrm{B} 1$ and $\mathrm{G} 2$, respectively) are much smaller than what is seen from the peak broadening in the XRD data. Then, it is straightforward to conclude that the $R$ interval is too narrow to appreciate the decrease in PDF peak intensities originating from the finite size of the diffracting domains.

(ii) Peak broadening as a function of the diffraction angle. In the wide-angle range, the broadening of the calculated peaks becomes comparable, or even narrower, than the experimental ones, which are definitely very sharp at smaller $2 \theta$. It is well known that the finite width of the distance distribution is a source of peak broadening that increases as a function of the diffraction angle (Ruland, 1965): this effect and the size broadening should fit the data over the whole $Q$ range. It looks likely that the distance distribution given by equation (21), or by similar formulas, holds only on a limited $R$ interval.

(iii) Peak shape. The peaks in the XRD data have a Lorentzian-like shape, pointing to a wide size distribution (Allegra, 1982). However, the calculated lines are Gaussianlike and, moreover, show the satellite ripples typical of a strictly sharp size distribution, pointing to the necessity, for a thorough structural characterization, of allowing for a suitable size distribution.

(iv) The (001) line at $11.25^{\circ} 2 \theta$ is split into two components, denoting different periodicities for the distance between basal planes. It is also evident that the simulated peak at $12.95^{\circ} 2 \theta$, characteristic of the c.c.p. arrangement, is shifted towards higher $2 \theta$ values with respect to the experimental data.

The conclusion of this analysis is that the long-range details of the structure are not captured correctly because the $R$-space model is tuned over a distance range that is much smaller than the actual particle size. In fact, the enlargement of the simulation interval to $60 \AA$, keeping the same parameters as determined by fitting in the 1-30 $\AA$ interval, shows that the difference plot is roughly twice as large beyond $30 \AA$ (see Fig. S4 in the supporting information).

\section{Concluding remarks}

This paper reports a real-space approach to the analysis of structural disorder in close-packed metals. The formalism detailed in Section 2 translates into $R$ space the Debye function analysis used to simulate powder diffraction patterns in $Q$ space. The $R$ and $Q$ approaches are in principle equivalent; the fundamental difference is that in $R$ space one can filter a given range of distances and then get local information about the sought-after structural features, while in $Q$ space all the distances contribute to the Fourier components of the diffraction lines.

A further relevant difference between the $R$ and $Q$ approaches is that the PDF signal broadening depends on the 
static and correlated disorder between pairs of atoms, while in $Q$ space the finite particle size also contributes to the broadening of the diffraction lines. Then, peak overlap and merging with the background in nanostructured samples could hamper the structural analysis in $Q$ space. On the other hand, the size effect in $R$ space can be observed by the decrease of the PDF intensity with increasing distance, so that the two sources of broadening (size and disorder) can be neatly discriminated in the $R$-space approach. In principle, the simulation of the small-angle contribution could also allow for a fine determination of the shape and size distribution of scattering domains (see the discussion related to Fig. 1), but this point requires further investigation. The PDF analysis carried out on simulated XRD data demonstrates that the $R$ approach can give reliable results on nanosized samples: in such cases the optimized stacking fault parameters can be determined also on a limited $R$ range. An indicative example of the issues involved in the case of overparameterization of the stacking faults model is given in Section 3.1, presenting the case of a Reichweite $s=4$ used for the analysis of an $s=3$ virtual sample. The optimized values of the stacking fault parameters indicate a fair capacity for convergence towards the 'true' structure, but a scan of parameter space shows the drawback of overparameterization.

We carried out a structural analysis on cobalt samples composed of large metallic particles, showing finer details of the diffraction lines and a PDF pattern extending over a wide range of distances. The $R$-space approach was integrated by a $Q$-space feedback. In the 1-30 $\AA$ interval the assessment of stacking faults yielded a very good fitting with a Reichweite $s=4$ model that, translated into $Q$ space, was able to simulate all the diffraction peaks corresponding to both the f.c.c. and h.c.p. packing. The $Q$ feedback, in contrast, demonstrated that the $R$-space approach failed at capturing the details of the size distribution of the crystallites, clearly due to the fact that the investigated $R$ range is much smaller than the average particle size, and that two phases are most likely present in the samples.

In conclusion, it is apparent that the $R$-space analysis can give a complete structural characterization of a disordered sample, if a suitable range of distances is taken into account. Then, the $R$-space approach could be particularly effective with nanostructured samples, where the distance range is limited by the actual sample size. On the other hand, the opportunity of filtering the range of investigated distances could be a new approach to the length-scale evaluation of structural disorder.

\section{Related literature}

Related literature cited in the supporting information: Gautschi (1970), Kölbig (1970).

\section{Acknowledgements}

We acknowledge the European Synchrotron Radiation Facility for provision of beamtime on beamline ID22 (proposal HC-2327). We thank Professor C. Meneghini (Università di Roma Tre) for friendly and useful discussion.

\section{References}

Allegra, G. (1982). Acta Cryst. A38, 863-867.

Bertolotti, F., Moscheni, D., Migliori, A., Zacchini, S., Cervellino, A., Guagliardi, A. \& Masciocchi, N. (2016). Acta Cryst. A72, 632-644.

Cervellino, A., Giannini, C. \& Guagliardi, A. (2006). J. Comput. Chem. 27, 995-1008.

Dmowski, W., Yin, H., Dai, S., Overbury, S. H. \& Egami, T. (2010). J. Phys. Chem. C, 114, 6983-6988.

Dupraz, M., Beutier, G., Rodney, D., Mordehai, D. \& Verdier, M. (2015). J. Appl. Cryst. 48, 621-644.

Egami, T. \& Billinge, S. J. L. (2003). Underneath the Bragg Peaks: Structural Analysis of Complex Materials, ch. 6. Pergamon Materials Series 7. Amsterdam: Elsevier.

Farrow, C. L. \& Billinge, S. J. L. (2009). Acta Cryst. A65, 232-239.

Gautschi, W. (1970). SIAM J. Numer. Anal. 7, 187-198.

Giacovazzo, C. (1992). Fundamentals of Crystallography, edited by C. Giacovazzo, ch. 3. Oxford University Press.

Gnanamani, E., Someshwar, N. \& Ramanathan, C. R. (2012). Adv. Synth. Catal. 354, 2101-2106.

Hamilton, W. C. (1965). Acta Cryst. 18, 502-510.

Hodeau, J.-L., Bordet, P., Anne, M., Prat, A., Fitch, A. N., Dooryhée, E., Vaughan, G. \& Freund, A. K. (1998). Proc. SPIE, 3448, 353-361.

Hong, X., Ehm, L., Zhong, Z., Ghose, S., Duffy, T. S. \& Weidner, D. J. (2016). Sci. Rep. 6, 21434.

Hosemann, R. \& Bagchi, S. N. (1962). Direct Analysis of Diffraction by Matter. Amsterdam: North-Holland.

Jagodzinski, H. (1949). Acta Cryst. 2, 340

Jeong, I., Proffen, T., Mohiuddin-Jacobs, F. \& Billinge, S. J. L. (1999). J. Phys. Chem. A, 103, 921-924.

Juhás, P., Davis, T., Farrow, C. L. \& Billinge, S. J. L. (2013). J. Appl. Cryst. 46, 560-566.

Kakinoki, J. (1967). Acta Cryst. 23, 875-885.

Kha, T. M., Schoenstein, F., Zighem, F., Nowak, S., Leridon, B., Jouini, N. \& Mercone, S. (2017). J. Magn. Magn. Mater. 422, 221-226.

Kölbig, K. S. (1970). Program C 335, CERN.

Li, W., Borkiewicz, O. J., Saubanère, M., Doublet, M.-L., Flahaut, D., Chupas, P. J., Chapman, K. W. \& Dambournet, D. (2018). J. Phys. Chem. C, 122, 23861-23866.

Longo, A. \& Martorana, A. (2008). J. Appl. Cryst. 41, 446-455.

Longo, A., Sciortino, L., Giannici, F. \& Martorana, A. (2014). J. Appl. Cryst. 47, 1562-1568.

Meng, Q., Guo, S., Zhao, X. \& Veintemillas-Verdaguer, S. (2013). J. Alloys Compd, 580, 187-190.

Mitra, G. B. \& Hadler, N. C. (1964). Acta Cryst. 17, 817-822.

Page, K., Proffen, T., Terrones, H., Terrones, M., Lee, L., Yang, Y., Stemmer, S., Seshadri, R. \& Cheetham, A. K. (2004). Chem. Phys. Lett. 393, 385-388.

Poulain, A., Sobczak, I. \& Ziolek, M. (2016). Cryst. Growth Des. 16, 5985-5993.

Pousthomis, M., Anagnostopoulou, E., Panagiotopoulos, I., Boubekri, R., Fang, W., Ott, F., Atmane, K. A., Piquemal, J.-Y., Lacroix, L.-M. \& Viau, G. (2015). Nano Res. 8, 2231-2241.

Proffen, Th. \& Billinge, S. J. L. (1999). J. Appl. Cryst. 32, 572-575.

Rocha, T. C. R., Martin, C., Kycia, S. \& Zanchet, D. (2009). AIP Conf. Proc. 1092, 102-106.

Ruland, W. (1965). Acta Cryst. 18, 992-996.

Sławiński, W. A., Zacharaki, E., Fjellvåg, H. \& Sjåstad, A. O. (2018). Cryst. Growth Des. 18, 2316-2325.

Sokalski, V., Laughlin, D. E. \& Zhu, J. (2011). J. Appl. Phys. 110, 093919.

Warren, B. E. (1969). X-ray Diffraction, ch. 13. New York: Dover.

Warren, B. E. \& Averbach, B. L. (1952). J. Appl. Phys. 23, 497.

Wilson, A. J. C. (1942). Proc. R. Soc. Lond. A, 180, 277-285.

Yu, Y., Cui, F., Sun, J. \& Yang, P. (2016). Nano Lett. 16, 3078-3084. 